\title{
Research on Design of Switching Power Supply Based on Mobile Base Station
}

\author{
CHEN Xuechang,a \\ ${ }^{1}$.Institute of Applied Electronics, Chongqing College of Electronic Engineering; Chongqing 401331, China \\ a.Email: pcboy2004@126.com
}

Keywords: Mobile Base Station, Switching Power Supply, Control Circuit

\begin{abstract}
With the rapid development of mobile communication service, the construction of mobile communication base station presents the trend of rapid development, the distribution of base station is more and more wide, more and more new requirements are put forward for the maintenance management of communication equipment power. According to the special environment and requirement of base station communication power supply, by using corresponding circuit control analysis and heat dissipation design, two double-pipe forward circuit parallel topologies, hot-plug interface design technology and non-hot-point design natural cooling design technology are adopted. Communication power module, the reliability of the power system is improved, the power module can also be hot plug, greatly improving the efficiency of power supply maintenance work, greatly reducing the average power system failure time, improve the economic operation of communications equipment, improve the mobile communication base station maintenance management level.
\end{abstract}

\section{Theoretical Introduction of Mobile Base Station Power Supply}

With the rapid development of mobile communications, the number of mobile base stations is increasing, and gradually from the city to the remote rural development, scattered in every corner of the life of mobile base stations, compared with the general communications room has its special working conditions. These special working conditions for mobile base stations for communications power equipment put forward higher requirements, mainly in the following areas:

The use of rural power supply mobile base station of its power supply quality can't be guaranteed, so the communication power supply can adapt to wide voltage fluctuations, generally more than $30 \%$. In addition, the mobile base station power grid over-voltage, lightning over-voltage more serious. Power should be reliable over-voltage and lightning protection measures. Many mobile base stations in the equipment put into operation early, often damp, high temperature, dust, etc., therefore require communication power with moisture, high temperature, dust and other measures ${ }^{[1]}$.

Mobile base station number, unattended, therefore require communication power supply easy maintenance, simple operation, with remote monitoring and strong fault diagnosis function, in order to ensure smooth communication, mobile base station communication power should have the missing phase running function. Mobile base station special working environment and working conditions determine the mobile base station communication power system with the special requirements for mobile base stations to provide power practice and lessons learned, we must improve the mobile base station communication power system reliability, maintainability and availability.

\section{Main Circuit Design of Switching Power Supply}

Switching power supply generally single-ended forward, single-ended fly back, double forward, dual single-ended forward, push-pull, half-bridge, full bridge topology. Single-ended forward, single-ended fly back, dual single-ended forward, push-pull switch pressure in the double input voltage above, it is easy to select the appropriate switch. In the push-pull and full-bridge topology, there may be one-way bias saturation, so that the switch tube damage, and half-bridge circuit 
because of the ability of automatic anti-unbalanced, so this problem does not occur. Double-tube forward and half bridge circuit switch pressure is only the maximum power supply voltage input, even if degree of use is $60 \%$, it is easy to select the appropriate switch. In the high-reliability engineering generally use these two types of circuit topology ${ }^{[2]}$.

The double-pipe forward converter has many advantages over the single-pipe forward converter, especially in the voltage stress, because each power device in the converter only needs to bear the power supply voltage, and in the single-tube forward converter, about twice the supply voltage, the cost of the switch is two. And it does not have the risk of a straight-through of the bridge arm as compared to a half-bridge or full-bridge converter. In this regard, we select the dual-transistor forward converter as the base station communication power of the main circuit topology. Fig. 1 shows main circuit structure of double-pipe forward converter.

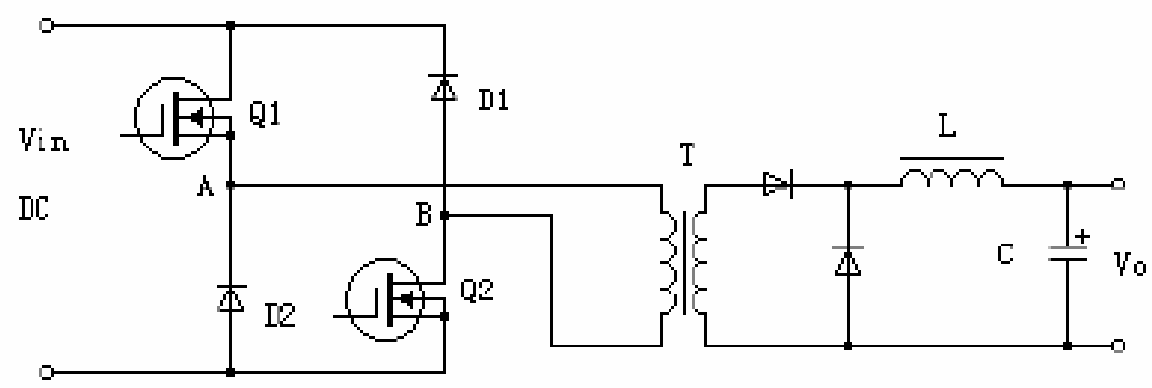

Fig.1. Main circuit structure of double-pipe forward converter

Three-phase three-wire system, there is no midline current problem; drawback is the use of active power factor correction to achieve the difficulties, the general use of passive power factor correction circuit (Figure 1), the use of three-phase power supply, power factor correction can be used active and passive, . Passive power factor correction circuit in the rectifier and capacitor in series between a filter inductor, the main advantage is simple, low cost, high reliability. The disadvantage is that it is difficult to get high power factor, performance and frequency, load, and input voltage changes ${ }^{[3]}$.

Three-phase input, passive power factor correction of the highest reliability, lowest cost, no midline current hidden drawback is that the power factor is not high, but fully meet the requirements of national standards. Switching power supply input circuit are mostly used rectifier plus capacitor filter circuit. In the input circuit closing moment, as the initial voltage on the capacitor will be zero to form a large instantaneous inrush current. For this end, the national standard on the communication power module switching machine overshoot amplitude, start the inrush current was strictly limited: switch overshoot amplitude required maximum peak does not exceed $10 \%$ of DC output setting; $150 \%$ of the current RMS. So we must be set in the switching power supply input circuit to prevent the impact of current soft-start circuit, and ensure normal and reliable switching power supply operation.

\section{Control Circuit Design of Switching Power Supply}

PWM switching regulator or steady-flow power supply is the basic working principle of the input voltage changes, internal parameter changes, external load changes, the control circuit is controlled by the difference between the signal and the reference signal for closed-loop feedback, adjust the main circuit switching device conduction pulse width, making the switching power supply output voltage or current are controlled and stable. Fig.2 shows control circuit design of switching power supply. 


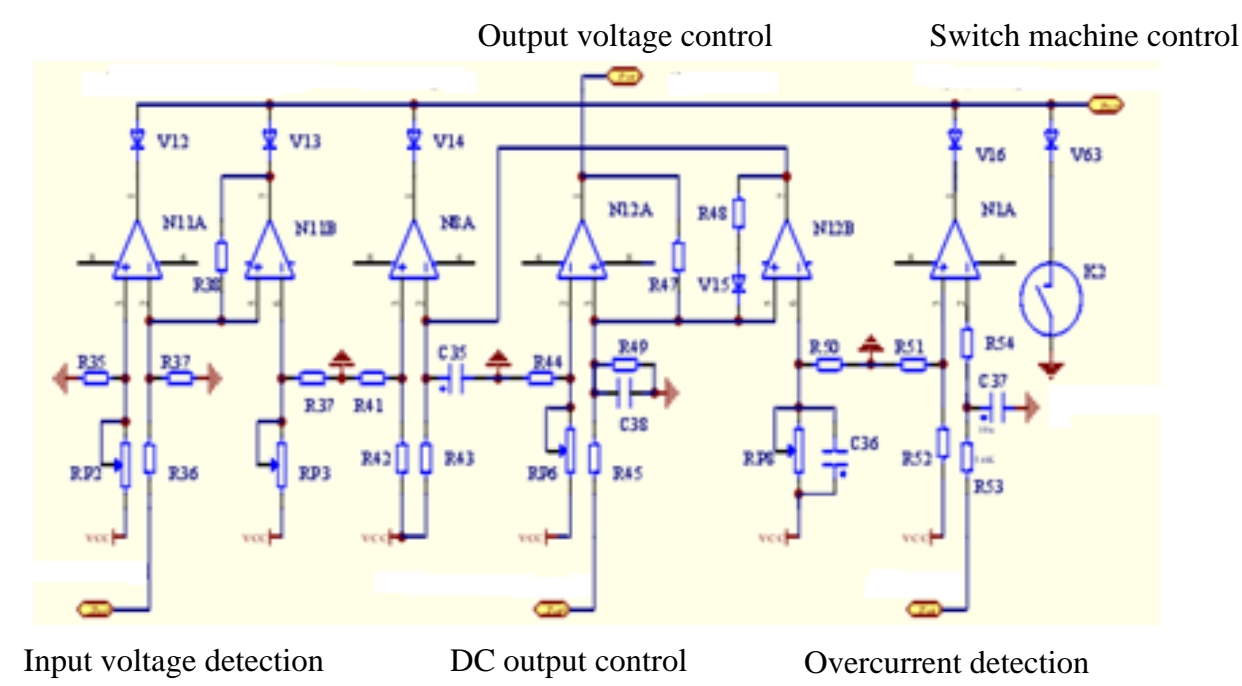

Fig.2.The control circuit design of switching power supply

PWM control mode is usually divided into two kinds of voltage-based controller and current-mode controller. Voltage-based controller can achieve voltage feedback control, requirements of stable output voltage. Current-based control method in the voltage closed-loop control, based on the increase in current feedback control, in addition to stable output voltage, there are the following advantages: When the current flows through the switch to a given value, achieve the switch automatically shut down by wave current limit. Transient closed-loop response is faster, the input voltage changes and changes in output load transient response are fast. Automatically eliminate the power frequency input rectified ripple voltage, can reduce the output filter capacitor. When multiple power modules are connected in parallel, the current-mode control design is simple. Based on the above analysis and comparison, using current-mode control and voltage-type control will greatly improve the dynamic performance of power modules to improve the reliability of the power supply module.

AC power supply module input, DC output and control signal lines collectively referred to as the power module interface. The current power interface connector uses a basic connector, which includes the AC input, DC output and control signal of the module. At present, the more advanced module installation method for hot-swappable, uninterruptible power supply system can be installed. Hot-swappable design refers to the power module has a module of online and offline diagnostic circuit, the power module can be inserted and pulled out, the module generally do not need to input and output switch. The technical requirements for power supply installation are low, reduced cost, and the maintenance time of the power supply system is shortened. In this regard, the design of the program selected hot-swappable ${ }^{[4]}$.

\section{Radiating design of Switching Power Supply}

The thermal design task of the base station communication power module is based on the basic principle of thermodynamics, choose the reasonable heat dissipation way and radiator, design the heat flow path as low as possible for the communication power supply module so that the heat of the semiconductor power device is dissipated as soon as possible, To achieve the communication power module within the electronic components in its allowable temperature range of work to ensure the reliability of the power supply module. At the same time should control the power module cost and size, improve the communication power module performance ratio.

Natural cooling can improve the reliability of the system is very favorable, especially for base station communication power, there are a lot of trouble to replace the fan. Taking into account the design using natural cooling as a communication power module cooling mode. Improve the communication power module reliable cooling measures base station communication power module with self-cooling design, the module's temperature rise will increase, which requires the power 
module radiator, module structure and power device installation should be reasonable, the module should not be obvious about hot spots, in order to avoid electronic devices working environment temperature is too high ${ }^{[5]}$.

In this regard, the design proposed the following solutions: select the radiator left plenty of margin, the semiconductor power devices are evenly distributed in the two radiators, the power module radiator no hot spots; the control circuit board and output capacitor board were put After the front panel and rear panel, with the hot radiator isolation, to ensure that the power supply module of electronic components at room temperature, improve the life of the power module; module design and system cabinet design consider the module cooling special wind road, the formation of heat sink module will form a convection heat dissipation in the duct. The heat of each module due to a dedicated cooling duct, without any affect each other.

\section{Conclusions}

With the continuous progress of the society, the business scope of the mobile communication industry is also developing constantly. It is very important to strengthen the communication coverage of nearby and remote areas. However, these areas have poor electricity supply and even no electricity supply, so guarantee the power supply is a big challenge in these base stations. The special environment and working condition of the mobile base station determines the special requirements of the mobile base station communication power system, combined with the practice and experience for the mobile base station power supply, the mobile communication power supply system intelligent put forward the mobile base station communication power. The modules are very important in the system, such as plug characteristics, no-load operation fault protection and natural cooling method of mobile base station power supply system.

\section{References}

[1] Sindona, A.Sortino,M.A. powerful and inexpensive supervision and control system for highly distributed Power Plants networks[C].INTELEC. Proceedings of high national IEEE Telecommunications Energy Conference.2010.5:126-128.

[2] Marques, A.B.Taranto, GN.Faleao, D. M.A knowledge-based system for supervision and control of regional voltage Profile and security [J].IEEE Transmit on Power Systems.2011.

[3] A. Delaille, M. Perrin, F. Huet, and L. Hernout, Study of the 'coup de fouet' of lead-acid cells as a function of their state-of-charge and state-of-health [ J]. Power Sources, vol. 158, no. 2, pp. 1019 1028, Aug. 2012.

[4] M.Guan, W.WEI,Y.Bao. A Monitoring System Based on Embedded Internet Technology for Embedded Devices. Computer Science and Software Engineering, 2008 International Conference on, 2008(4):10-29.

[5] Shuang Hua Yang, Chengwei Dai, Roger P.Knott. Remote maintenance of control system performance over the Internet. Control Engineering Practice, 2007, 15(5): 286-317. 